\title{
Thyroid Fine-Needle Aspiration Cytology Practice in Korea
}

Yoon Jin Cha $\cdot$ Ju Yeon Pyo

SoonWon Hong · Jae Yeon Seok ${ }^{1}$

Kyung-Ju Kim ${ }^{2} \cdot$ Jee-Young $\mathrm{Han}^{3}$

Jeong Mo Bae ${ }^{4} \cdot$ Hyeong Ju Kwon ${ }^{5}$

Yeejeong $\mathrm{Kim}^{6} \cdot$ Kyueng-Whan Min

Soonae $\mathrm{Oak}^{8} \cdot$ Sunhee Chang ${ }^{9}$

Department of Pathology, Gangnam Severance Hospital, Yonsei University College of Medicine,

Seoul; 'Department of Pathology, Gachon

University Gil Medical Center, Incheon;

Department of Pathology, Yeungnam University

College of Medicine, Daegu; ${ }^{3}$ Department of

Pathology, Inha University Hospital, Incheon;

${ }^{4}$ Department of Pathology, Seoul National

University Hospital, Seoul; ${ }^{5}$ Department of

Pathology, Wonju Severance Christian Hospital,

Yonsei University Wonju College of Medicine,

Wonju; ${ }^{6}$ National Health Insurance Service

Ilsan Hospital, Goyang; ${ }^{7}$ Department of

Pathology, Hanyang University Guri Hospital,

Hanyang University College of Medicine, Guri;

${ }^{8}$ Department of Pathology, IIsin Christian

Hospital, Busan; 'Department of Pathology,

Inje University IIsan Paik Hospital, Goyang, Korea

Received: August 12, 2017

Revised: September 16, 2017

Accepted: September 25, 2017

Corresponding Author

SoonWon Hong, MD, PhD

Department of Pathology, Gangnam Severance

Hospital, Yonsei University College of Medicine,

211 Eonju-ro, Gangnam-gu, Seoul 06273, Korea

Tel: +82-2-2019-3540

Fax: +82-2-3463-2103

E-mail: soonwonh@yuhs.ac
We reviewed the current status of thyroid fine-needle aspiration cytology (FNAC) in Korea. Thyroid aspiration biopsy was first introduced in Korea in 1977. Currently, radiologists aspirate the thyroid nodule under the guidance of ultrasonography, and cytologic interpretation is only legally approved when a cytopathologist makes the diagnosis. In 2008, eight thyroid-related societies came together to form the Korean Thyroid Association. The Korean Society for Cytopathology and the endocrine pathology study group of the Korean Society for Pathologists have been updating the cytologic diagnostic guidelines. The Bethesda System for Reporting Thyroid Cytopathology was first introduced in 2009, and has been used by up to $94 \%$ of institutions by 2016 . The average diagnosis rates are as follows for each category: I (12.4\%), II (57.9\%), III (10.4\%), IV (2.9\%), V (3.7\%), and VI (12.7\%). The malignancy rates in surgical cases are as follows for each category: I (28.7\%), II (27.8\%), III (50.6\%), IV (52.3\%), V (90.7\%), and VI (100.0\%). Liquidbased cytology has been used since 2010 , and it was utilized by $68 \%$ of institutions in 2016 . The categorization of thyroid lesions into "atypia of undetermined significance" or "follicular lesion of undetermined significance" is necessary to draw consensus in our society. Immunocytochemistry for galectin-3 and BRAF is used. Additionally, a molecular test for BRAF in thyroid FNACs is actively used. Core biopsies were performed in only $44 \%$ of institutions. Even the institutions that perform core biopsies only perform them for less than $3 \%$ of all FNACs. However, only $5 \%$ of institutions performed core biopsies up to three times more than FNAC.

Key Words: Bethesda; Fine needle aspiration cytology; Thyroid neoplasms; Korea
In this review, we surveyed the current status of thyroid fineneedle aspiration cytology (FNAC) in Korea and briefly described the history of FNAC in Korea. The multiple topics covered in this review include thyroid cytology sample collectors and interpreters, cytotechnician training programs, preparation methods for thyroid cytology samples, staining of thyroid cytology samples, thyroid cytology reporting systems and data distribution, the use of "atypia of undetermined significance" and "follicular lesion of undetermined significance" (AUS/FLUS) as diagnostic categories, the thyroid cytology audit program, correlation between cytology and histology, external quality assurance for thyroid FNAC, and the status of ancillary testing including core biopsy.

The 2016 survey project was designed to create a short communication that was compiled by a limited number of members of the Korean Society for Cytopathology (KSC). The 2012 survey $^{1}$ is more comprehensive than this 2016 survey, but we compared 2012 and 2016 data in this paper. We asked the KSC to administer the survey to the 210 institutions under its quality control. 
Thirty-eight institutions responded to this survey within 3 weeks. The survey items included thyroid cytology sample collectors and interpreters, preparation methods for thyroid cytology samples, thyroid cytology reporting systems and data distribution, the use of AUS/FLUS categorization, correlation between cytology and histology, and the status of core biopsy. Twelve among the 38 responders answered distribution data for each class of the Bethesda reporting system. Eight institutions also sent correlation data between cytology and histology. Graphs and statistical analysis of Student's $t$ test for categorical diagnosis rates were constructed using Excel Software. Categorical variables were expressed as percentage, frequency, and range. Differences were considered statistically significant at $\mathrm{p}<.05$.

\section{BRIEF HISTORY OF THYROID FINE-NEEDLE ASPIRATION CYTOLOGY}

In Europe, thyroid FNAC was introduced in the 1950 s, ${ }^{2}$ but in the United States, it began to be more actively used in the 1970s. ${ }^{3}$ Thyroid aspiration biopsy was first introduced in Korea by a physician in $1977 .{ }^{4}$ As for the introduction of FNAC to pathologists, it was started by a pathologist who was experienced in aspiration cytology from cytopathology training in Europe in the 1980s. ${ }^{5}$ In the early stages of use, aspirations were initially performed by a radiologist who had undergone European training, and the aspirates were sent to pathologists for interpretation. At that time, clinicians directly interpreted the aspiration cytology slide; however, with the development of quality control and insurance coverage, the frequency of clinician's interpretation has decreased. Initially, aspiration cytology was performed only on palpable lesions of superficial organs, but with the development of imaging system, it has become possible to accurately locate and aspirate nonpalpable lesions with very high diagnostic accuracy. Therefore, FNAC has become prevalent at the majority of medical institutions. ${ }^{6}$ In 2006, pathologists created management guidelines for patients with thyroid nodules and thyroid cancer as a mainstay of the Korean Endocrine Society. ${ }^{7}$ As a result, the Endocrine Pathology Study Group was created in 2007. In 2008, eight thyroid-related societies made up the Korean Thyroid Association. The main goal of the Endocrine Pathology Study Group and the KSC is to update the cytologic diagnostic guideline. ${ }^{8}$ According to recent data, 196,000 cases of thyroid FNAC are performed each year in Korea, accounting for $60 \%$ of all FNACs performed. ${ }^{9}$ Due to a perceived over-diagnosis of thyroid cancer, the performance of FNAC as a whole decreased by $19.6 \%$ in 2015 compared to
2014, based on the data of our survey.

\section{THYROID CYTOLOGY SAMPLE COLLECTORS AND INTERPRETERS}

In the early stages of FNAC, endocrinologists, surgeons, radiologists, and pathologists aspirated palpable thyroid nodules and interpreted them using a Giemsa stain. After ultrasonography was introduced, radiologists began to aspirate thyroid nodules more than any other specialists. Now, national insurance covers the aspiration fee only when radiologists aspirate the thyroid nodule by guided ultrasonography. While radiologists perform the aspiration, legal standards dictate that a diagnosis can only be made by a cytopathologist. Therefore, cytologic interpretation has been carried out at more than 200 cytology laboratories throughout the country, all of which are subject to quality control by the KSC.

\section{CYTOTECHNICIAN TRAINING PROGRAM}

In 1981, the Korean National Medical Center, under the auspices of the World Health Organization (WHO), launched a nationwide education program for cytotechnicians to work as cytology screeners. This is considered to be the first systematic cytologic education program in Korea. Under the direction of the WHO, this course was planned to increase early detection of cervical cancer, the most frequent cancer at the time, and it was a first step toward a national cancer eradication project (National Cancer Control Program) planned by the Health and Social Affairs Department (present Health and Welfare Department) in 1978. The need for systematic cytologic screening and education was widely supported by the government. To establish the Cytology School of the National Medical Center's Pathology Department, a Swedish cytotechnician, Barbro Nilsson, came to Korea in 1981 as a WHO adviser who worked as the primary cancer screening personnel (cytotechnician) educator. The WHO supported this adviser by providing educational equipment such as microscopes and lanterns; other healthcare professionals, like Swedish doctors Nils, who was the then president of the International Academy of Cytology (IAC), and Stormby, also donated slide teaching materials and textbooks for gynecological cytology. At that time, cytotechnician education was carried out at the National Medical Center with the main purpose of improving the quality of cytopathology examination conducted at university hospitals and general hospital nationwide. Under the direction of anatomic pathologists, we selected 10 cytotechnicians 
who were in charge of cytology screening, nine of whom attended their first training course in November 1981, and in the second course of the following year, we again selected other 10 cytotechnicians and educated. ${ }^{6}$

Thyroid FNAC is included in the cytotechnician education program; however, participation in cytology screening depends on the institutional policy.

\section{PREPARATION OF THYROID CYTOLOGY SAMPLES}

At the time of introduction, FNACs were analyzed with air-dried Giemsa stain; since 2000, they have been read using an alcoholfixed conventional smear with Papanicolaou staining and cell block preparation. Currently, a few institutions still use the airdried Giemsa stain.

Liquid-based cytology (LBC), which decreases the rate of cell paucity, was introduced for FNAC preparation despite some inherent disadvantages such as unfamiliar cellular morphology. Since 2010, LBC had been widely adopted and was used by $44 \%$ of institutions in 2012 and by $68 \%$ in 2016. In the 2012 survey, LBC alone was not sufficient for diagnosis, so 22 of 33 institutions $(67 \%)$ used LBC combined with conventional methods. In 2016, the number of institutions adding a supplemental method dropped to 12 of 23 (52\%). This reduction can be attributed to increased experience in LBC interpretation. ${ }^{1}$ For FNAC spec- imens, ThinPrep (45.0\%) was most commonly used, followed by SurePath (33.6\%), EASY Prep (12.9\%), and Huro Path (4.5\%). ${ }^{9}$

\section{STAINING OF THYROID CYTOLOGY SAMPLES}

Staining was initially performed using air-dried Giemsa stain, but the Papanicolaou stain has become predominant. However, some institutions still use hematoxylin and eosin or Giemsa stains.

\section{THYROID CYTOLOGICAL REPORTING SYSTEMS AND DATA DISTRIBUTION}

Until the Bethesda System for Reporting Thyroid Cytopathology (TBSRTC) appeared in 2008, ${ }^{10}$ thyroid FNAC was diagnosed in a variety of ways; at some institutions, it was diagnosed using descriptive terms, in the same format as a pathology diagnosis.

The diagnosis of thyroid FNAC was made based mainly on the Papanicolaou Society Guidelines, ${ }^{11}$ as a result, the Korean Endocrine Society published management guidelines for patients with thyroid nodules and thyroid cancer in 2006 . $^{7}$

After that, the Endocrine Pathology Study Group was created in 2007. In 2008, eight thyroid-related societies made up the Korean Thyroid Association, and TBSRTC was introduced to Korea in 2009. The Endocrine Pathology Study Group and the KSC have been updating the cytologic diagnostic guideline

Table 1. Cytologic diagnosis rates according to TBSRTC

\begin{tabular}{|c|c|c|c|c|c|c|}
\hline \multirow{2}{*}{ Institution } & \multicolumn{6}{|c|}{ Category } \\
\hline & 1 & $\|$ & III & IV & V & $\mathrm{VI}$ \\
\hline 1 & 8.6 & 61.7 & 6.1 & 5.5 & 3.4 & 14.7 \\
\hline 3 & 8.0 & 61.0 & 2.3 & 0.6 & 4.5 & 23.6 \\
\hline 4 & 15.4 & 51.5 & 16.5 & 0.5 & 4.8 & 11.4 \\
\hline 5 & 8.1 & 60.0 & 11.8 & 1.1 & 2.9 & 16.2 \\
\hline 6 & 11.2 & 56.9 & 7.6 & 1.0 & 4.3 & 19.0 \\
\hline 7 & 20.1 & 34.1 & 21.9 & 0.7 & 5.7 & 17.4 \\
\hline 8 & 20.3 & 44.5 & 18.1 & 2.0 & 3.0 & 12.2 \\
\hline 9 & 32.6 & 61.5 & 3.2 & 0.2 & 1.9 & 0.6 \\
\hline 10 & 2.9 & 86.5 & 2.7 & 0.0 & 0.6 & 7.3 \\
\hline 11 & 0.0 & 74.9 & 9.7 & 0.0 & 3.4 & 12.0 \\
\hline 12 & 5.5 & 50.0 & 6.6 & 22.3 & 6.8 & 8.9 \\
\hline Average (\%) & 12.4 & 57.9 & 10.4 & 2.9 & 3.7 & 12.7 \\
\hline Range (\%) & $0-32.6$ & $34.1-86.5$ & $2.3-21.9$ & $0-22.3$ & $0.6-6.8$ & $0.6-23.6$ \\
\hline Average $(\%)^{12}$ & 12.9 & 59.3 & 9.6 & 10.1 & 2.7 & 5.4 \\
\hline Range $(\%)^{12}$ & $1.8-23.6$ & $39.0-73.8$ & $3.0-27.2$ & $1.2-25.3$ & $1.4-6.3$ & $2.0-16.2$ \\
\hline$p$-value ${ }^{a}$ & .790 & .723 & .992 & .053 & .385 & .008 \\
\hline
\end{tabular}

TBSRTC, the Bethesda System for Reporting Thyroid Cytopathology.

aStatistical analysis of student's t test for categorical diagnosis rate between this survey data and reference data were constructed from Excel Software. Differences for which $p<.05$ were considered significant. 
based on TBSRTC. ${ }^{8}$ According to the survey conducted in 2012, 60 of the 74 responding institutions (80\%) used TBSRTC, ${ }^{1}$ while the 2016 survey showed that 94\% (34/36) are using TBSRTC.

Twelve institutions reported distribution data according to class in the Bethesda reporting system. Eight institutions also sent correlation data between cytology and histology.

The categorical diagnosis rate of each institution by TBSRTC is as follows: I (12.4\%), II (57.9\%), III (10.4\%), IV (2.9\%), V (3.7\%), and VI (12.7\%), as shown in Table 1 and Fig. 1.

The average value is not very unique compare to worldwide reports, ${ }^{12}$ but some categories show significant differences between this present survey data and Bongiovanni's meta-analysis data. ${ }^{12}$ Categories 1, 2, 3, and 5 showed rates similar to Bongiovanni's data. However, category 6 was significantly higher in this Korean survey than in Bongiovanni's data ( $12.7 \%$ vs $5.4 \%, \mathrm{p}=.008$ ). Additionally, category 4 was lower in this Korean data than in Bongiovanni's data, but the difference was not statistically significant $(2.9 \%$ vs $10.1 \%, \mathrm{p}=.053)$.

This survey is limited in that the proportion of referral hospitals is higher than the distribution of nationwide laboratories in Korea.

\section{USE OF ATYPIA OF UNDETERMINED SIGNIFICANCE AND FOLLICULAR LESION OF UNDETERMINED SIGNIFICANCE}

In this survey, we looked at how to appropriately use the AUS/FLUS terminology of TBSRTC. In 66\% of institutions, the two terms are used to indicate the same lesion, although most use only the term AUS. Only two institutions use the term AUS/FLUS.

In the remaining $34 \%$ of institutions, the two terms are used differently. AUS is used in cases with nuclear atypia, and FLUS is used in cases where there is architectural atypia. This is not consistent with the original intent of TBSRTC. In the case of AUS/FLUS, subcategorized studies are often reported by multiple institutions. ${ }^{13}$ Confusion exists surrounding this term, and it is necessary to establish consensus on the proper use of this term.

\section{THYROID CYTOLOGY AUDIT PROGRAM: CORRELATION BETWEEN CYTOLOGY AND HISTOLOGY}

The quality control system is managed by each institution, and reports on the quality control of thyroid FNACs have been published since 1996..$^{14,15}$

Accuracy was assessed based on malignancy rates using cytohistological correlations, but some reports assessed the accuracy of each specific diagnostic entity. There are about 12 papers addressing this accuracy, of which about four demonstrated accuracy using TBSRTC., ${ }^{1,16-18}$

The malignancy rates for overall cytologic diagnoses are as follows for each category: I (1.8\%), II (0.7\%), III (6.3\%), IV (19.1\%), V (51.9\%), and VI (63.5\%) (Fig. 2A). The malignancy rates for surgical cases are as follows for each category: I (28.7\%), II (27.8\%), III (50.6\%), IV (52.3\%), V (90.7\%), and VI (100.0\%) (Fig. 2B).

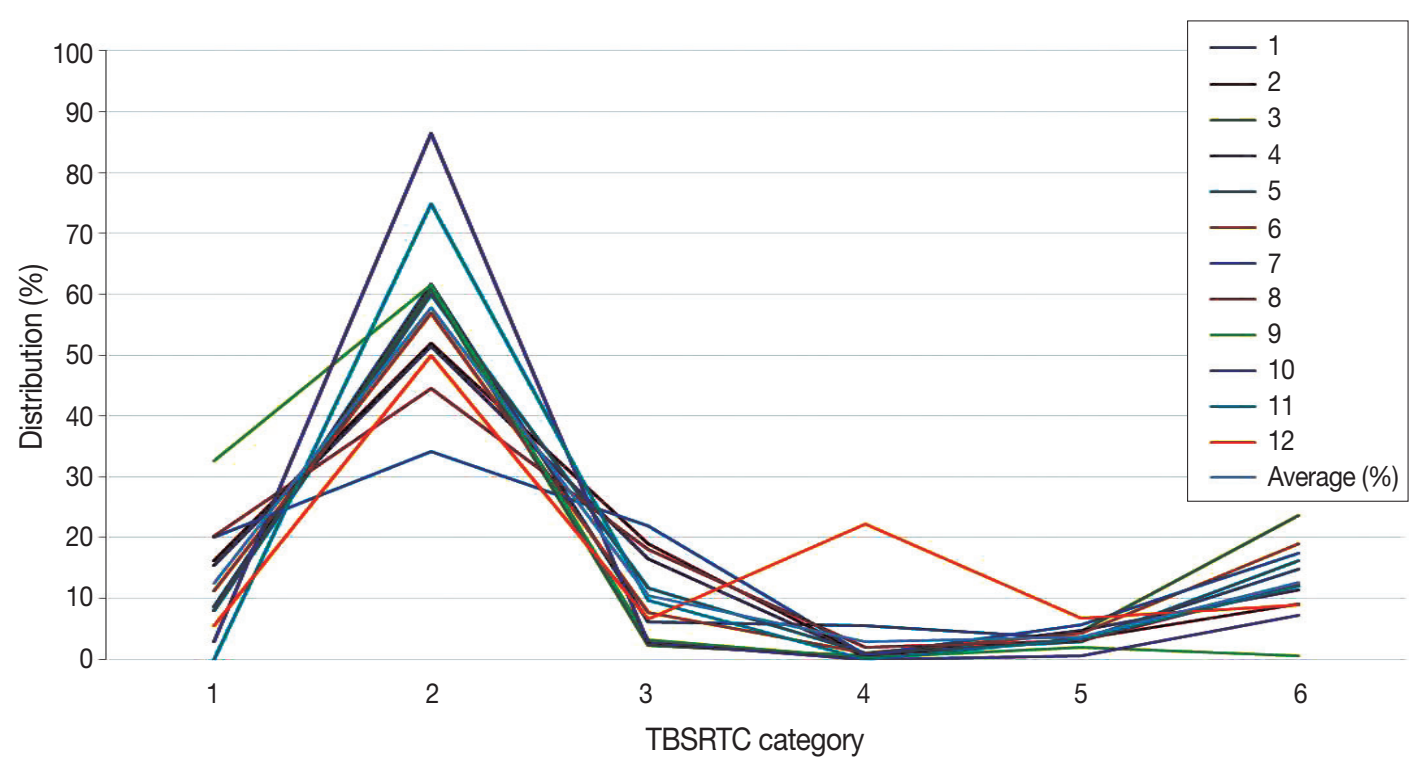

Fig. 1. The diagnosis rate of each institution by the Bethesda System for Reporting Thyroid Cytopathology (TBSRTC) category. 


\section{EXTERNAL QUALITY ASSURANCE FOR THYROID FINE-NEEDLE ASPIRATION CYTOLOGY}

Since 1995, the KSC has been in charge of quality control of fine needle aspiration smears, and the quality control findings have been reported since $1999 .{ }^{6}$ Quality evaluation by the Korean Society of Pathologists was added in 2007, and each institute is making efforts to improve the quality of thyroid FNAC, as well as overall cytopathology. ${ }^{19}$

\section{THE STATUS OF ANCILLARY TESTING INCLUDING CORE BIOPSY}

Immunocytochemical staining could theoretically be used to
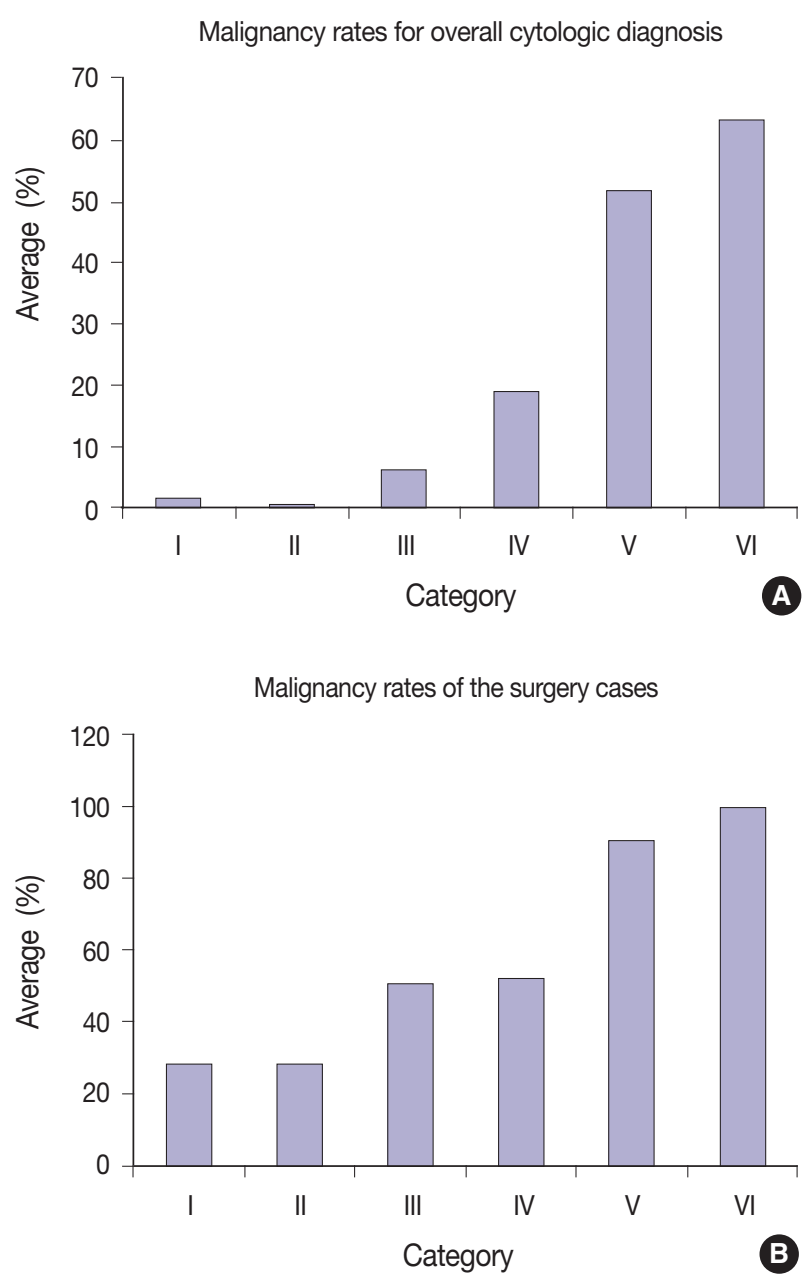

Fig. 2. (A) The malignancy rates for overall cytologic diagnoses are as follows for each category: I (1.8\%), II (0.7\%), III (6.3\%), IV (19.1\%), V (51.9\%), and VI (63.5\%). (B) The malignancy rates for surgical cases are as follows for each category: I (28.7\%), II (27.8\%), III (50.6\%), IV (52.3\%), V (90.7\%), and VI (100.0\%). make cytologic diagnoses, but immunocytochemical targets useful for thyroid cytology have not been identified. There have been many reports of galectin-3 being applied to cytologic diagnosis since 2008, but the results have been controversial. ${ }^{20-22}$

$\beta$-Catenin, CXCL12, and other rare immunocytochemical stains are also used for cytology. There are current reports of immunocytochemistry for BRAF, and some institutions use it for actual analysis. ${ }^{23-26}$ Immunostaining for cytologic examination is also covered by insurance.

Since 2010, a molecular pathology approach, including the BRAFV600E mutation, has been utilized, and various molecular pathological studies are underway. However, only the BRAF test is actively used at present and approved by insurance. ${ }^{27-30}$

In recent years, core biopsies have been performed mainly in Korea and Italy. However, core biopsies were performed in only 17 of the 36 institutions (44\%) in this survey. Even institutions that perform core biopsy used the procedure in less than $3 \%$ of FNACs.

However, at two of these institutions, core biopsy was performed up to three times more than FNAC. There are 45 articles on core biopsies written by Korean authors, most of which are reported by radiologists who prefer core biopsy.

These radiologists asked pathologists to establish a standardized classification for core biopsy compatible with the TBSRTC. Pathologists agreeing with this idea have published a paper that standardizes core biopsy readings. ${ }^{31}$

In Korea, pathologists are interpreting both pathology and cytopathology. Therefore, for pathologists, histologic interpretation of a core biopsy per se is easier than the cytological interpretation of FNAC if difficulties or side effects accompanying the core biopsy procedure are not considered.

However, considering that we often cannot distinguish adenomatous hyperplasia from follicular neoplasm, even if we examine all surgical specimens, it is controversial to say that it is more accurate to make this distinction with core biopsy than with FNAC.

\section{CONCLUSION}

Currently, radiologists aspirate thyroid nodules under the guidance of ultrasonography, and legal standards dictate that a diagnosis can only be made by a cytopathologist.

The TBSRTC was used by up to 94\% of institutions in 2016. The average diagnosis rates are as follows for each category: I (12.4\%), II (57.9\%), III (10.4\%), IV (2.9\%), V (3.7\%), and VI $(12.7 \%)$. The malignancy rates in surgical cases are as follows for 
each category: I (28.7\%), II (27.8\%), III (50.6\%), IV (52.3\%), V (90.7\%), and VI (100.0\%).

Since 2010, LBC has been used and was implemented by $68 \%$ of institutions in 2016.

It is necessary to draw consensus on the use of the terms AUS and FLUS.

Immunocytochemistry is used in galectin- 3 and BRAF assays. For molecular tests in thyroid FNACs, BRAF is actively used. Core biopsies are performed only rarely in a few institutions.

\section{Conflicts of Interest}

No potential conflict of interest relevant to this article was reported.

\section{Acknowledgments}

We would like to thank the participants of members of the Korean Society of Cytopathology: Dr. Young Lyun Oh, Samsung Medical Center, Sungkyunkwan University School of Medicine, Seoul; Dr. Ju Hie Lee, Kyung Hee University Medical Center, Seoul; Dr. Ae Ri An, Chonbuk National University Hospital, Jeonju; Dr. Hee Sung Kim, Chung-Ang University College of Medicine, Seoul; Dr. Jin Hee Sohn, Kangbuk Samsung Hospital, Sungkyunkwan University School of Medicine, Seoul; Dr. Yosep Chong, College of Medicine, The Catholic University of Korea, Seoul; Dr. Dong Eun Song, Asan Medical Center, University of Ulsan College of Medicine, Seoul; Dr. Hyun Ju Lee, Soonchunhyang University Cheonan Hospital, Soonchunhyang University College of Medicine, Cheonan; Dr. Tae Sook Hwang, Konkuk University School of Medicine, Seoul; Dr. Mi Kyung Shin, Hallym University Kangnam Sacred Heart Hospital, Seoul; Dr. Kang Min Han, Dongguk University Ilsan Hospital, Ilsan; Yeong-Seon Hong, Good Samsun Hospital, Busan; Dr. Myoung Jin Ju, Presbyterian Medical Center, Jeonju; Dr. Hye-Sun Kim, Cheil General Hospital \& Women's Healthcare Center, Dankook University College of Medicine, Seoul; Dr. Yoon Hee Jin, Seongnam Central Hospital, Seongnam; Dr. Dongyoul Choi, GC Labs, Youngin, Dr. Byung Doo Lee, Seegene Medical Foundation, Seoul; Dr. Yoo Duk Choi, Chonnam National University Medical School, Gwangju; Dr. Song-Yi Choi, Chungnam National University School of Medicine, Daejeon; Dr. Min Gyoung Pak, Dong-A University College of Medicine, Busan; Dr. Ji-Young Choe, Hallym University Sacred Heart Hospital, Anyang; Dr. Hyojin Kim, Seoul National University Bundang Hospital, Seongnam; Dr. Songmi Noh, CHA Gangnam Medical Center, CHA University, Seoul; and Dr. Hyunju Yoo, Daerim Saint
Mary’s Hospital Thyroid Center, Seoul, Korea.

\section{REFERENCES}

1. Kim M, Park HJ, Min HS, et al. The use of the Bethesda System for Reporting Thyroid Cytopathology in Korea: a nationwide multicenter survey by the Korean Society of Endocrine Pathologists. J Pathol Transl Med 2017; 51: 410-7.

2. Lipton RF, Abel MS. Aspiration biopsy and the thyroid in evaluation of thyroid dysfunction. Am J Med Sci 1944; 208: 736-42.

3. Crile G Jr, Hawk WA Jr. Aspiration biopsy of thyroid nodules. Surg Gynecol Obstet 1973; 136: 241-5.

4. Lee MH. Thyroid biopsy. Korean J Med 1977; 20: 731-6.

5. Park HS. Cytohistopathologic comparative study of aspiration biopsy cytology from various sites. Korean J Cytopathol 1991; 2: 8-19.

6. Lee K. The history of the Korean Society for Cytopathology. Seoul: The Korean Society for Cytopathology, 2006; 15-9.

7. Kim WB, Kim TY, Kwon HS, et al. Management guidelines for patients with thyroid nodules and thyroid cancer. J Korean Endocr Soc 2007; 22: 157-87

8. Yi KH, Lee EK, Kang HC, et al. 2016 Revised Korean Thyroid Association management guidelines for patients with thyroid nodules and thyroid cancer. Int J Thyroidol 2016; 9: 59-126.

9. Oh EJ, Jung CK, Kim DH, et al. Current cytology practices in Korea: a nationwide survey by the Korean Society for Cytopathology. J Pathol Transl Med 2017 Sep 27 [Epub]. https://doi.org/10.4132/ jptm.2017.08.11.

10. Cibas ES, Ali SZ; NCI Thyroid FNA State of the Science Conference. The Bethesda System For Reporting Thyroid Cytopathology. Am J Clin Pathol 2009; 132: 658-65.

11. Guidelines of the Papanicolaou Society of Cytopathology for the examination of fine-needle aspiration specimens from thyroid nodules. The Papanicolaou Society of Cytopathology Task Force on Standards of Practice. Diagn Cytopathol 1996; 15: 84-9.

12. Bongiovanni M, Spitale A, Faquin WC, Mazzucchelli L, Baloch ZW. The Bethesda System for Reporting Thyroid Cytopathology: a meta-analysis. Acta Cytol 2012; 56: 333-9.

13. Jung YY, Jung S, Lee HW, Oh YL. Significance of subcategory atypia of undetermined significance/follicular lesion of undetermined significance showing both cytologic and architectural atypia in thyroid aspiration cytology. Acta Cytol 2015; 59: 370-6.

14. Kwon KH, Jin SY, Lee DW. A study of usefulness of fine needle aspiration cytology of the thyroid lesions. Korean J Cytopathol 1996; 7: 111-21.

15. Park KM, Ko IH. Diagnostic accuracy of fine needle aspiration cytology in thyroid lesions: analysis of histologically confirmed 153 cases. 
Korean J Cytopathol 1996; 7: 122-33.

16. Park JH, Yoon SO, Son EJ, Kim HM, Nahm JH, Hong S. Incidence and malignancy rates of diagnoses in the bethesda system for reporting thyroid aspiration cytology: an institutional experience. Korean J Pathol 2014; 48: 133-9.

17. Lee YB, Cho YY, Jang JY, et al. Current status and diagnostic values of the Bethesda system for reporting thyroid cytopathology in a papillary thyroid carcinoma-prevalent area. Head Neck 2017; 39: 269-74

18. Lee K, Jung CK, Lee KY, Bae JS, Lim DJ, Jung SL. Application of Bethesda system for reporting thyroid aspiration cytology. Korean J Pathol 2010; 44: 521-7.

19. The Korean Society of Pathologist. The History of the Korean Society of Pathologists 2006 2015. Seoul: The Korean Society of Pathologist, 2016; 20-1.

20. Jung CK, Lee A, Jung ES, Choi YJ, Jung SL, Lee KY. Split sample comparison of a liquid-based method and conventional smears in thyroid fine needle aspiration. Acta Cytol 2008; 52: 313-9.

21. Kim ES, Lim DJ, Lee K, et al. Absence of galectin-3 immunostaining in fine-needle aspiration cytology specimens from papillary thyroid carcinoma is associated with favorable pathological indices. Thyroid 2012; 22: 1244-50.

22. Gweon HM, Kim JA, Youk JH, et al. Can galectin-3 be a useful marker for conventional papillary thyroid microcarcinoma? Diagn Cytopathol 2016; 44: 103-7.

23. Koo JS, Jung W, Hong SW. Cytologic characteristics and betacatenin immunocytochemistry on smear slide of cribriform-morular variant of papillary thyroid carcinoma. Acta Cytol 2011; 55: 13-8.
24. Jung YY, Park IA, Kim MA, Min HS, Won JK, Ryu HS. Application of chemokine CXC motif ligand 12 as a novel diagnostic marker in preoperative fine-needle aspiration biopsy for papillary thyroid carcinoma. Acta Cytol 2013; 57: 447-54.

25. Lee SR, Yim H, Han JH, et al. VE1 antibody is not highly specific for the BRAF V600E mutation in thyroid cytology categories with the exception of malignant cases. Am J Clin Pathol 2015; 143: 437-44.

26. Kim YH, Yim H, Lee YH, et al. Evaluation of the VE1 antibody in thyroid cytology using ex vivo papillary thyroid carcinoma specimens. J Pathol Transl Med 2016; 50: 58-66.

27. Kwak JY, Kim EK, Kim JK, et al. Dual priming oligonucleotidebased multiplex PCR analysis for detection of BRAFV600E mutation in FNAB samples of thyroid nodules in BRAFV600E mutationprevalent area. Head Neck 2010; 32: 490-8.

28. So YK, Son YI, Park JY, Baek CH, Jeong HS, Chung MK. Preoperative $B R A F$ mutation has different predictive values for lymph node metastasis according to tumor size. Otolaryngol Head Neck Surg 2011; 145: $422-7$.

29. Lee SE, Hwang TS, Choi YL, et al. Molecular profiling of papillary thyroid carcinoma in Korea with a high prevalence of BRAFV600E mutation. Thyroid 2017; 27: 802-10.

30. Kim Y, Choi KR, Chae MJ, et al. Stability of DNA, RNA, cytomorphology, and immunoantigenicity in residual ThinPrep specimens. APMIS 2013; 121: 1064-72.

31. Jung CK, Min HS, Park HJ, et al. Pathology reporting of thyroid core needle biopsy: a proposal of the Korean Endocrine Pathology Thyroid Core Needle Biopsy Study Group. J Pathol Transl Med 2015; 49: 288-99. 